\title{
STRONGLY ANNULAR FUNCTIONS WITH SMALL COEFFICIENTS, AND RELATED RESULTS
}

\author{
D. D. BONAR, F. W. CARROLL ${ }^{1}$ AND PAUL ERDÖS
}

\begin{abstract}
A technique of Bagemihl and Seidel is applied to two problems in annular functions. It is shown that there exists a strongly annular function with Maclaurin coefficients tending to zero, and that there exist annular functions that are far from being strongly annular.
\end{abstract}

0. Introduction. We show that there is a function

$$
f(z)=\sum_{\nu=0}^{\infty} a_{\nu} z^{\nu}, \quad|z|<1
$$

such that

$$
\lim _{\nu \rightarrow \infty} a_{\nu}=0
$$

and

$$
\sup _{0<r<1} \min _{|z|=r}|f(z)|=\infty \text {. }
$$

A function $f$, holomorphic in the unit disk $D$ (briefly, $f \in \mathcal{H}(D)$ ), is said to be strongly annular if $(0.3)$ holds; an $f$ in $\mathcal{H}(D)$ is annular if

$$
\lim _{n \rightarrow \infty} \min \left\{|f(z)|: z \in J_{n}\right\}=\infty
$$

for some sequence of Jordan curves $J_{n}$ in $D$ with 0 in their interiors. An example of an annular function for which (0.2) holds was known previously [4, p. 100], [2, p. 21].

While it is known that not every annular function is strongly annular [3], one might speculate that every annular function enjoys some of the special properties of the strongly annular functions. For example, given an annular function $f$, can the $\left\{J_{n}\right\}$ satisfying $(0.4)$ always be chosen so that the sequence of lengths $l\left(J_{n}\right)$ remains bounded? Can the $\left\{J_{n}\right\}$ be chosen so that the ratio of the distances to $|z|=1$ from the closest and farthest points of $J_{n}$ is bounded away from zero as $n$ increases? In $\S 2$, we construct a counterexample to these conjectures.

Both constructions make use of a technique of Bagemihl and Seidel [1, pp. 188-190].

Recieved by the editors May 6, 1977.

AMS (MOS) subject classifications (1970). Primary 30A72; Secondary 30A10.

1 The second author acknowledges support from the National Science Foundation. 
1. Strongly annular functions with small Maclaurin coefficients. Let $H(D)$ be provided with the topology of uniform convergence on compact subsets of $D$. We use the methods of [1] to obtain the following lemma.

LEMMA. Let $\Re$ be a family of functions holomorphic in the closed unit disk. Suppose that, given any number $M>0$ and any neighborhood $\mathfrak{K}$ of 0 in $H(D)$, there is a function $g$ in $\mathscr{B} \cap \mathcal{N}$ such that $|g(z)|>M$ on $|z|=1$. Then there is a sequence $\left\{f_{k}\right\}$ in $\mathscr{B}$ such that the function

$$
f(z)=\sum_{k=1}^{\infty} f_{k}(z), \quad|z|<1,
$$

is strongly annular.

Proof. Choose $f_{1}$ in $\mathscr{B}$ so that $\left|f_{1}(z)\right|>1$ for $|z|=1$, then choose $r_{1}$, $0<r_{1}<1$, so close to 1 that the inequality holds on $|z|=r_{1}$. Next, choose $f_{2}$ in $\mathscr{B}$, so that: (i) $\left|f_{2}(z)\right|<2^{-2}$ in $|z| \leqslant r_{1}$ and $\left|f_{1}(z)+f_{2}(z)\right|>1$ on $|z|=r_{1}$, and (ii) $\left|f_{2}(z)\right|>2+\left|f_{1}(z)\right|$ on $|z|=1$. Choose $r_{2}, r_{1}<r_{2}<1$, so that the last inequality continues to hold on $|z|=r_{2}$. Continue choosing the functions $f_{k}$ and the numbers $r_{k}$, inductively, in the obvious way.

THEOREM 1. There exists a strongly annular function (0.1) such that (0.2) holds. More explicitly, $f$ is of the form (1.1), each $f_{k}$ being a polynomial; the coefficients are small and noninterfering:

$$
\begin{aligned}
& \text { (i) } f_{k}(z)=\sum_{\nu} \alpha(k, \nu) z^{\nu}, \\
& \text { (ii) }|\alpha(k, \nu)| \leqslant 1 / k, \\
& \text { (iii) } \alpha(k, \nu) \alpha(j, \nu)=0 \text { for } \nu=0,1, \ldots \text {, whenever } j \neq k .
\end{aligned}
$$

Let $\delta$ be the operator on the set of nonconstant complex polynomials defined by

$$
(\delta P)(z)=P(z) P\left(z^{d+1}\right), \quad d=\text { degree of } P,
$$

and let $\delta^{p}=\delta\left(\delta^{p-1}\right), p=2,3 \ldots$ We consider the particular polynomial $Q(z)=1-z+z^{2}+z^{3}+z^{4}$. One may verify that we have

$$
\left|Q\left(e^{i \theta}\right)\right|^{2}=5+2 \cos 2 \theta+2 \cos 4 \theta=\frac{11}{4}+4\left(\cos 2 \theta+\frac{1}{4}\right)^{2},
$$

so that the minimum modulus of $Q(z)$ on $|z|=1$ is $\mu=\sqrt{11} / 2 \doteq 1.66$. It is clear from (1.3) that the coefficients of $\delta^{p} Q$ are \pm 1 , and that its minimum modulus on $|z|=1$ is at least $\exp \left(2^{p} \log \mu\right)$.

DEFINITION. $\mathscr{B}_{1}$ is the sequence of polynomials

$$
g_{p}(z)=p^{-1} z^{m(p)} \delta^{p} Q(z), \quad p=1,2, \ldots,
$$

where $m(1)=0$ and $m(p+1)$ is one greater than the degree of $g_{p}$.

Proof of Theorem 1. Clearly the $g_{p}$ have small, noninterfering coefficients in the sense of (1.2). For $|z| \leqslant r$, we have

$$
\left|g_{p}(z)\right| \leqslant r^{m(p)} p^{-1}(1-r)^{-1},
$$


while on $|z|=1$, we have

$$
\left|g_{p}(z)\right| \geqslant p^{-1} \exp \left(2^{p} \log \mu\right) .
$$

Hence, the sequence $\mathscr{B}_{1}$ satisfies the hypotheses of the Lemma, and we can extract a subsequence $f_{k}=g_{p(k)}$ such that (1.1) is strongly annular.

\section{Functions far from strongly annular.}

THEOREM 2. There exists an annular function $f$ with the following property: If $\left\{J_{n}\right\}$ is any sequence of Jordan curves about 0 in $D$ for which (0.4) holds, then $l\left(J_{n}\right)$ approaches infinity as $n$ increases.

Proof. Choose $0<r_{1}<r_{2}<\cdots<1$. For each $n$, form a closed Jordan curve $I_{n}$ in $D$ which coincides with $|z|=r_{n}$ in the left semidisk, while in the right semidisk it is a perturbation of $|z|=r_{n}$ by a sinusoidal function of large frequency and small amplitude. These are chosen so that $l\left(I_{n}\right)$ is greater than $n$ and $I_{n}$ lies in the interior of $I_{n+1}$. The set $N\left(n, \varepsilon_{n}\right)$ of points of $D$ that lie less than $\varepsilon_{n}$ from $I_{n}$ is open, and we may choose $\varepsilon_{n}$ so small that for each Jordan curve $J$ about 0 that lies in $N\left(n, \varepsilon_{n}\right)$, we have $l(J)>n$. We require further that $N\left(n, \varepsilon_{n}\right) \cap N\left(n+1, \varepsilon_{n+1}\right)$ is empty.

For $n=1,2, \ldots$, we define a compact set $K_{n}$. It is the portion of the region between $I_{n}$ and $I_{n+1}$ that lies in the closed right semidisk and meets neither $N\left(n, \varepsilon_{n}\right)$ nor $N\left(n+1, \varepsilon_{n+1}\right)$. The set $K_{n}$ does not disconnect the plane.

Let $f_{1}(z)=2$ for all $z$. Suppose that, for some $n \geqslant 1$, we have found an entire function $f_{n}$ such that

$$
\begin{aligned}
& \left|f_{n}(z)\right|>j \text { for all } z \text { on } I_{j} \text { and for } j=1, \ldots, n, \\
& \left|f_{n}(z)\right|<1 \text { for all } z \text { in } \bigcup_{j=1}^{n-1} K_{j} .
\end{aligned}
$$

We then define an entire function $\eta_{n}(z)$ that has small modulus on $I_{n}$ (and hence in $I_{n}$ ), approximates $-f_{n}(z)$ on $K_{n}$, and has large modulus on $I_{n+1}$; such a function exists (cf. Remark 1). We choose the tolerances so that we have

$$
\begin{aligned}
& \left|f_{n}(z)+\eta_{n}(z)\right|>j \text { for all } z \text { on } I_{j}, j=1, \ldots, n+1, \\
& \left|f_{n}(z)+\eta_{n}(z)\right|<1 \text { for } z \text { in } \cup_{j=1}^{n} K_{j},
\end{aligned}
$$

and so that, if $f_{n+1}=f_{n}+\eta_{n}$, the sequence $\left\{f_{n}\right\}$ converges almost uniformly in the unit disk. The limit function $f$ is annular, and has modulus at most 1 on $\cup K_{j}$. Hence, each sequence $\left\{J_{n}\right\}$ for which $(0.4)$ holds meets only finitely many of the $K_{j}$, so that the lengths $l\left(J_{n}\right)$ must grow without bound.

REMARK 1. We add a few words about the existence of $\eta_{n}$. Take $g_{n}(z)=0$ on $J_{n}$ and its interior and $g_{n}(z)=-f_{n}(z)$ on $K_{n}$. By Runge's theorem, some entire function $h_{n}$ approximates $g_{n}$ on these two sets. Let $\psi_{n}$ be the continuous extension of a conformal map of the interior of $J_{n+1}$ onto $|w|<1$, and let $M$ be a number larger than $n+1+\max \left\{\left|f_{n}(z)+h_{n}(z)\right|:|z| \leqslant 1\right\}$. For $k$ sufficiently large, the function $M \psi_{n}^{k}$ has modulus $M$ on $J_{n+1}$ but is small on $J_{n} \cup K_{n}$. Approximate $M \psi_{n}^{k}$ by an entire function $\varphi_{n}$, and take $\eta_{n}=h_{n}+\varphi_{n}$.

REMARK 2. Instead of the sequence $|z|=r_{n}$, one may use a sequence 
$\left|z-a_{n}\right|=r_{n}$, with $r_{n}$ increasing to 1 and $a_{n}$ decreasing to zero, so that the circles do not intersect, and so that

$$
\lim _{r \rightarrow \infty} \frac{1-\left(r_{n}+a_{n}\right)}{1-\left(r_{n}-a_{n}\right)}=0 .
$$

If the $\varepsilon_{n}$ are taken small enough, the construction will give a function $f$ that is far from strongly annular in an additional sense. That is, for each sequence $\left\{J_{n}\right\}$ for which $(0.4)$ holds, the ratio of the distances to $|z|=1$ from the closest point of $J_{n}$ and from the farthest point approaches zero.

\section{REFERENCES}

1. F. Bagemihl and W. Seidel, Some boundary properties of analytic functions, Math. Z. 61 (1954), 186-199.

2. D. D. Bonar, On annular functions, VEB Deutscher Verlag Wiss., Berlin, 1971.

3. D. D. Bonar and F. W. Carroll, Not every annular function is strongly annular, J. Reine Angew. Math. 273 (1975), 57-60.

4. J. E. Littlewood, Lectures on the theory of functions, Oxford Univ. Press, London, 1944.

Department of Mathematical Sciences, Denison University, Granville, Oho 43023

Department of Mathematics, Oho State University, Columbus, Oho 43210

Mathematical Institute, Hungarian Academy of Sciences, Budapest, Hungary 